\title{
New Technique Combining the Tone Reservation Method with Clipping Technique to Reduce the Peak-to-Average Power Ratio
}

\author{
Hajar Abdelali ${ }^{1}$, Smail Bachir $^{2}$, and Mohamed Oumsis ${ }^{3}$ \\ ${ }^{1}$ Lims Laboratory, Faculty of Sciences Dhar Mahraz, Sidi Mohamed Ben Abdellah University of Fez, Morocco \\ ${ }^{2}$ Xlim Laboratory, UMR CNRS 7252, University of Poitiers, France \\ ${ }^{3}$ Lrit Laboratory, Unit Associated With CNRST, URAC 29, University Mohamed V, Rabat, Maroc
}

\begin{abstract}
Article Info
Article history:

Received Dec 26, 2017

Revised Jul 3, 2018

Accepted Jul 29, 2018

Keyword:

OFDM System

PAPR Reduction

WiMAX IEEE 802.16e

Tone Reservation

Clipping

Conjugate Gradient

ABSTRACT

Nonlinear distortions and impairments appear in multicarrier signal with high fluctuations when amplified by a Radio Frequency Power Amplifier (RF PA). This article is a contribution to improving the Tone Reservation (TR) method dedicated to reducing fluctuations in the Orthogonal Frequency Division Multiplexing (OFDM) modulation. This method exploits the null sub-carriers in communications standards in order to generate a correction signal used to reduce the Peak-to-Average Power Ratio (PAPR) with nevertheless limited performances due to the reduced number of these sub-carriers. For this purpose, our contribution is to combine the TR method with the Clipping (CL) method to improve the PAPR reduction without significantly impacting the quality of transmissions. We also show the gain provided by this strategy compared to the use of classical methods. Experiments using a simulated example on a complete WiMax 802.16e transmitter have been made in order to investigate the PAPR reduction performances on presence of the non-linear Power Amplifier model based on gain compression response and phase distortion.
\end{abstract}

Copyright (c) 2018 Institute of Advanced Engineering and Science. All rights reserved.

\section{Corresponding Author:}

Hajar Abdelali,

LIMS Laboratory,

Faculty of Sciences Dhar Mahraz,

Sidi Mohamed Ben Abdellah University of Fez, Morocco.

abdelali.hajar@usmba.ac.ma

\section{Introduction}

OFDM technique [1] is a very popular modulation method and tends to become the most widespread multicarrier modulation in recent radio communication systems, such as Wireless Local Area Network (WLAN), Worldwide Interoperability for Microwave Access (WiMax) and DVB. Today, it was chosen for the fourth Generation (4 G) mobile communication systems, where the Long Term Evolution Advanced (LTE-A) standard allows broadband services with a theoretical transmission rate up to $100 \mathrm{Mbps}$. The advantages of OFDM modulation are: its optimal spectral congestion, its flexibility in the allocation of the sub-carriers and its simplicity of implementation using Fourier transforms at the transceiver.

However, it presents some disadvantages and drawbacks such as; OFDM system generates high power peaks in time-domain signal, termed as Peak to Average Power Ratio (PAPR) [2] results in nonlinear distortions when amplified by the Radio Frequency Power Amplifier (RF PA) [3],[4]. These distortions lead to a inband spectral regrowth and out-of-band spectral regrowth that result in degraded communication metrics like 
Adjacent Channel Power Ratio (ACPR), Error Vector Magnitude (EVM) and Bit Error Rate (BER).

In literature, there are several approaches for PAPR reduction like CL [5],[6], Clipping-Filtering (CF) [7],[8],[9], Selective Mapping (SLM) [9],[10],[11], Partial Transmit Sequence (PTS) [9],[10],[12], Block Coding [13], Tone Injection [14] and TR [15],[16]. These methods differ in their principle and implementation, but also in their drawbacks: some of them add side information decreasing data rate and/or bandwidth like PTS, others sacrifice BER performances for the benefit of PA efficiency such as CL and CF while some others are efficient but too difficult to implement like SLM.

In this paper, our contribution called (TRCL) is a new method based on the combination of two methods to reduce PAPR, TR [15],[16] with Conjugate Gradient Optimization algorithm [17],[18] and CL [5],[6]. These two methods present a certain complementarity: the TR method based on the addition of signal is one of the methods having the property of downward compatibility and improving the performance of EVM and BER due to their low degradation and their low intake of supplementary computational complexity, but its performance remains limited because of the reduced number of free subcarriers, while the CL is very simple of those known this day and theoretically allows an unlimited clipping of the signal but unfortunately with a degradation of all the communication criteria and a decrease in performance caused by a loss of some information.

Despite its limitations, this is the TR method that is preferred in practice because it requires no modification the receiver. In [19], the author proposed the use of the TR method based on the conjugate gradient algorithm in order to reduce the value of PAPR and improve the speed of convergence. In this paper, we are going to describe and study our TRCL method and compare it with other existing methods TR and CL. We will see below how our new method improves the performance in terms of PAPR reduction and the communication criteria EVM and BER.

The study of the performances in terms of reduction of the PAPR is carried out on the standard WiMax - IEEE 802.16e [20]. To study the impact of this association, simulations in the presence of a Solid State Power Amplifiers (SSPA) modeled by a nonlinearity of the gain and phase, are carried out in order to evaluate the quality of the transmissions.

The paper will be organized as follows: section 2 will present the Problem position, while section 3 , will discuss two methods of PAPR reduction: Clipping and Tone Reservation based on the Conjugate Gradient Optimization Algorithm, and present the proposed technique based on the conjunction of CL and TR (TRCL). In section 4 , we present the simulation results of our new method TRCL compared to the TR and the CL. Finally, we conclude, in section 5 .

\section{Problem Position}

To transmit a signal, the OFDM signal model (figure 1) divides at first a frequency range into several independent orthogonal sub-carriers which have the same width of frequency band. The representation of an OFDM signal can then be written:

$$
c(k)=\frac{1}{\sqrt{N}} \sum_{k=0}^{N-1} C_{k} e^{j\left(\frac{2 \pi n k}{N}\right)}, 1 \leq n \leq N
$$

where $C_{k}$ is the data symbols with $k=0, \cdots, N-1$ is the index of the sub-carrier, $C=\left[C_{0}, C_{1}, \cdots, C_{N-1}\right]$ indicate the carrier vector data and $\mathrm{N}$ is the number of sub-carriers. Subsequently, it applies the algorithm of the inverse Fourier Transform (IFFT) on this OFDM signal by the Following equation:

$$
x=Q \cdot X
$$

where $\mathrm{Q}$ is the matrix of transformation IFFT of size $\mathrm{N}$.

From equation 1, when all the sub-carriers are weighted at high level, the constructive sum needs to appear peaks in the temporal domain. This establishes the major inconvenience of the OFDM systems [1]. To measure these fluctuations, the PAPR, defined as a highest peak power to average power of one OFDM symbol, such as:

$$
\operatorname{PAPR}(x)_{d B}=10 \cdot \log _{10} \frac{\max _{0 \leq t \leq T}|x(t)|^{2}}{E\left(|x(t)|^{2}\right)}
$$




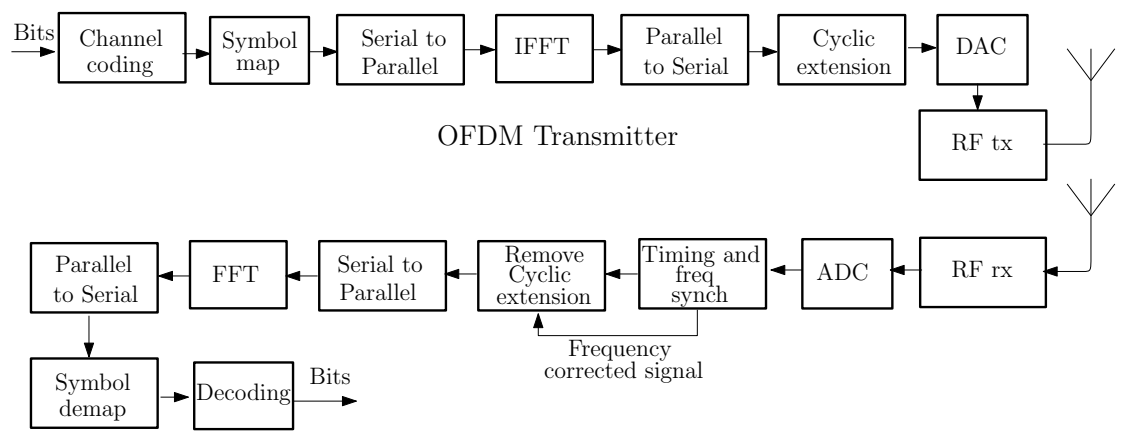

OFDM Receiver

Figure 1. Model of OFDM transmitter and receiver for WIMAX

In literature, we can find several techniques for PAPR reduction [2] which present some improvements but at the cost of many inconveniences. For example, the CL method has in theoretical unlimited performances in term of PAPR reduction but its application induces systematically errors of transmission (like increasing BER and/or Out-Of-Band distortion, etc.). These drawbacks can be avoided using the TR method, but the use of only nul subcarriers allows about 2 to $4 \mathrm{~dB}$ of improvement. Here, we propose new technique combining TR and CL methods to improve performances by avoiding the disadvantages of each one. and CL:

As we previously noted, we will cite the advantages and the inconveniences of the two techniques TR

- TR : this technique is one of the methods having the property of downward compatibility and improves the performance of "EVM" and "BER" due to their low degradation and their low intake of supplementary computational complexity but it is limited by a restricted number of free subcarriers in the standard, complexity of implementation, etc.

- CL : this technique is very simple and has an unlimited performances in term of PAPR reduction but its application induces systematically degradation of all transmission criteria (BER and/or Out-of-Band distortion)

\section{Proposed Technique}

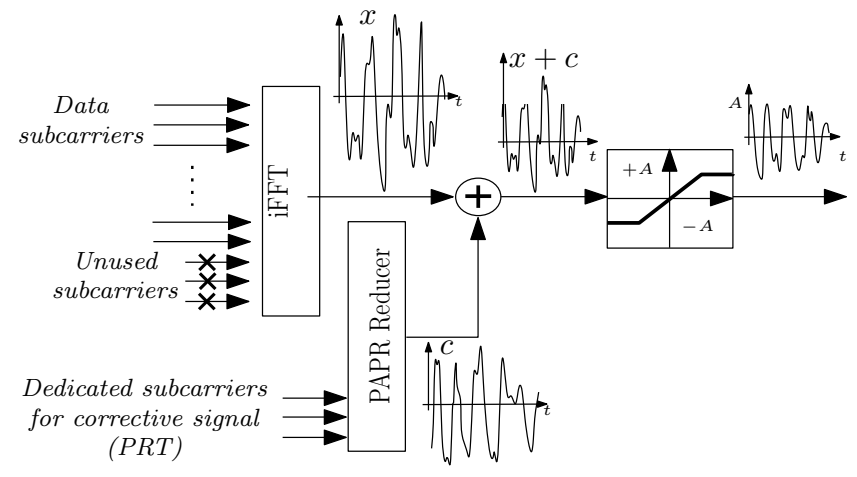

Figure 2. The combination of both techniques TR and CL (TRCL).

Here, we will investigate the possibilities of combining both techniques TR and CL (figure 2). These two techniques can be complementary because the TR allows to reduce the majority of peaks in an OFDM symbol. Still a few samples of the symbol that cannot be reduced by this method and which will be affected by the non-linearities of the circuits such as the power amplifier. In this case, we propose to apply the CL method to avoid any exceeding the clipping value. 


\subsection{Clipping}

CL [5],[6] is the simplest method of those known this day which allows reducing the value of PAPR. The objective of this method is to clip all points whose amplitude exceeds a predetermined threshold $A$ to reduce the power variation. This procedure is repeated until no point exceeds this threshold. Equation 4 shows the principle of clipping. The simplicity of this method has an impact on the signal reception performance will be reduced because the information removed by clipping are lost.

$$
f(x)= \begin{cases}A \cdot \operatorname{sign}(x(t)) & \text { if }|x(t)|>A \\ x(t) & \text { otherwise }\end{cases}
$$

\subsection{Tone Reservation}

TR [15],[16] is the most recent method based on the addition of a signal to the original signal to reduce the PAPR. The Useful sub-carriers are reserved for the broadcast of the original signal and the sub-carriers not used are exploited to generate the signal of cancellation of peak without loss of flow of data and Reduced the PAPR. The generation of the temporal signal for the reduction of the PAPR by process TR was formulated as problem of convex optimization which requires a high computational cost of the order of $\mathcal{O}\left(N_{r} N^{2} L\right)$, where $N_{r}$ is the number of reserved carriers, $N$ the number of carriers of the OFDM and $L$ is the over-sampling factor.

To solve this problem, there are four iterative optimization algorithms, but in this paper we are going to concentrate on one optimization algorithm: Conjugate Gradient. It is an iterative method just like the method of the gradient, but it uses an algorithm so that the direction of research is optimal. This direction is determined by the gradient at the point $X_{n}$ and the gradient of the previous point $X_{n-1}$ [17]. For the first iteration, the chosen direction is the negative gradient:

$$
d_{0}=-g_{0}
$$

At the $k+1^{t h}$ iteration, the point $X_{n+1}$ is chosen by finding the minimum of the function in the direction previously determined to reduce the number of iterations.

$$
X_{k+1}=X_{k}+\alpha_{k} \cdot d_{k}
$$

$\alpha_{k}$ is the conjugate gradient step, and $d_{k}$ represents the direction of conjugate gradient. The next direction is determined by the previous direction. This method involves combining the previous direction $d_{k-1}$ with the gradient at the point $X_{k}$ to calculate the new direction:

$$
d_{k}=-g_{k}+\rho_{k} \cdot d_{k-1}
$$

There are several versions of the method of the combined gradient, they vary by the way the constant $\rho_{k}$ is calculated. Here is the method of calculation of Fletcher-Reeves [21]:

$$
\rho_{k}=\frac{g_{k}^{T} \cdot g_{k}}{g_{k-1}^{T} \cdot g_{k-1}}
$$

$\rho_{k}$ represents then the report between the standard to the square of the current gradient and the standard to the square of the previous gradient. Here is the method of calculation of Polak-Ribire [22]:

$$
\rho_{k}=\frac{\left(g_{k}-g_{k-1}\right)^{T} \cdot g_{k}}{g_{k-1}^{T} \cdot g_{k-1}}=\frac{\Delta g_{k-1}^{T} \cdot g_{k}}{g_{k-1}^{T} \cdot g_{k-1}}
$$




\subsection{New TRCL Method}

The number of null subcarriers in a given standard is limited, the TR method reduces the majority of peaks in an OFDM symbol, but always remains some samples whose amplitude is high and which will consequently be affected by the non-linearities of the circuits like the power amplifier. In this case, we propose to apply, after the TR, the CL method in order to avoid any exceeding of the A threshold.

Therefore, the first phase is to reduce the PAPR, using the TR method which calculates the correction signal c, from the null subcarriers, according to the Conjugate Gradient algorithm which is used to reduce the convergence time of the optimization algorithm.

The second phase consists in applying a clipping to the same threshold $\mathrm{A}$ as the previous phase in order to eliminate the remaining peaks

\section{Simulation Results}

In this section, we present the experimental evaluation results of our new method TRCL with the standard IEEE 802.16e or "WiMAX" [20]. The following table 1 represents the WiMAX parameters used in simulations to improve the PAPR reduction of OFDM signals.

Table 1. The IEEE 802.16 parameters used in simulation

\begin{tabular}{ll}
\hline Name of the parameter & value \\
\hline System & OFDM \\
Modulation & 64QAM \\
Num of Sub-carriers & 512 \\
Sub-carriers Datas & 384 \\
Sub-carriers Pilots & 42 \\
Sub-carriers GI & 86 \\
$P A P R_{\text {-target }}$ & 2.17 \\
\hline
\end{tabular}

This standard is a broadband wireless technology of the fourth generation allowing data transmission on long distances (dozens of kilometers until 50km) at high speed $70 \mathrm{Mbits} / \mathrm{s}$ and with an optimal security. The systems based on the $802.16 \mathrm{e}$ standard is called "Mobile Wimax". The communication by this standard can be realized on Line Of Sight (LOS) or Not Line Of Sight (NLOS). The number of carriers for WIMAX 802.16e can vary between 2048, 1024, 512 and 128, and there are three types of subcarriers for OFDM: Subcarrier data, Pilot subcarriers and Subcarriers care (or subcarriers zeros).

The distribution of these sub-carriers varies according to the mode of allocation FUSC (Fully Used Sub Channelization) and PUSC (Partially Used Sub Channelization) and the nature of the link DL (Downlink) or UL (Uplink): these two modes differ by the fact of total or partial use of channels and depend on the need for the users.

In this paper, the simulation is carried out for 512 subcarriers and we consider the Mode sub-channel FUSC in the downlink DL by using a Fast Fourier Transform (FFT) (figure 3).

\subsection{PAPR reduction}

To make an evaluation on the movement of a signal, there are several criteria to make it, among these criteria we find the PAPR and the CCDF. As we have already seen previously that the PAPR is the report between the maximum power and the averages power of a temporal signal. However, for a better movement evaluation of a signal, we use the second parameter CCDF which is a Complementary Cumulative Distribution Function of the movement on an interval of fixed time. Thus CCDF is the probability that the value of PAPR is superior to a fixed value.

$$
C C D F=\operatorname{Pr}\left(P A P R \geq P A P R_{0}\right)
$$




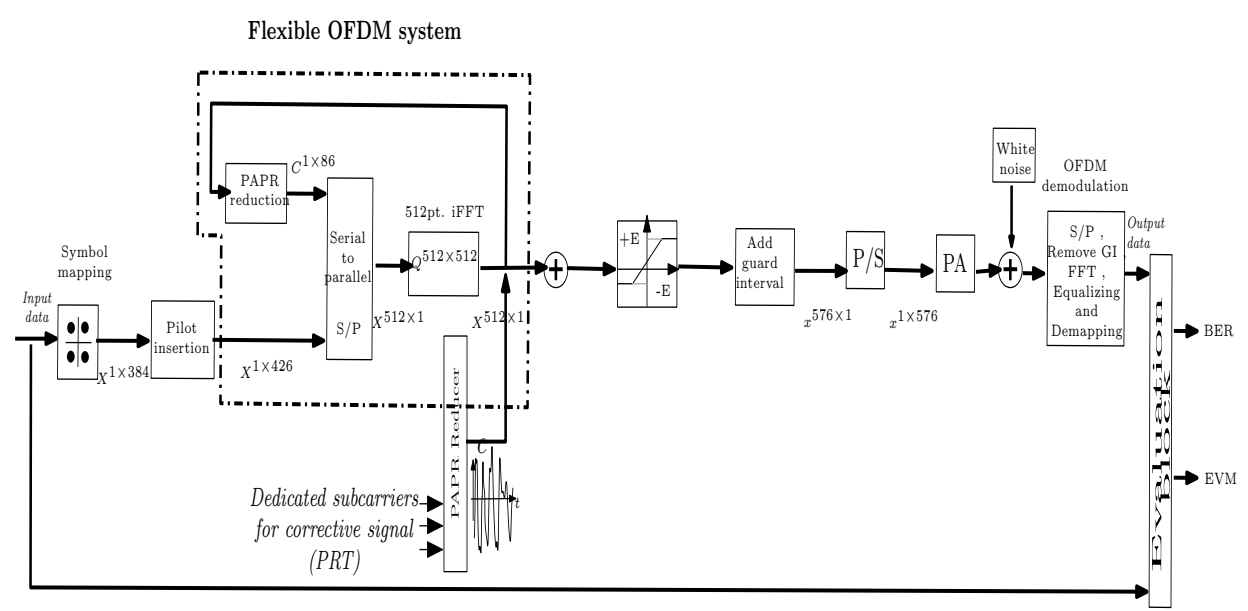

Figure 3. OFDM data symbol generation and TRCL scheme for 802.16e standard

For that purpose, for any signal, the dynamics of this signal will always be superior or equal to $0 \mathrm{~dB}$ thus the value of PAPR will also have a probability superior or equal to $0 \mathrm{~dB}$. The more the CCDF parameter has a low value, the more the probability of having a high PAPR value tends to 0 .

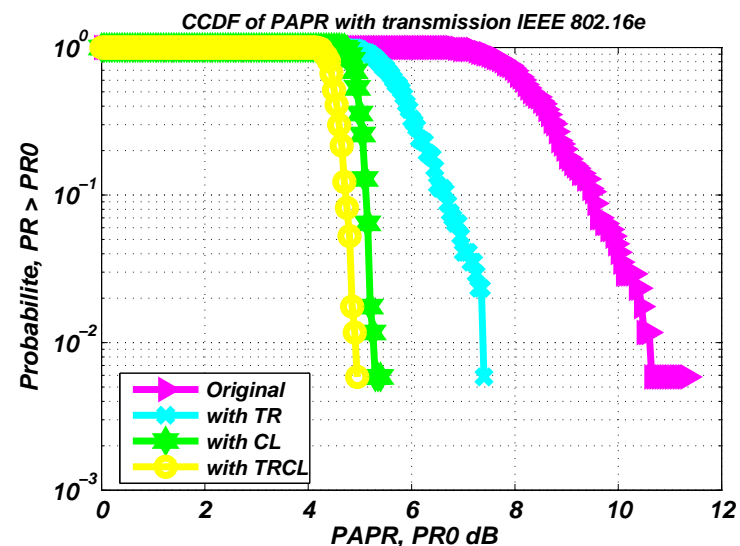

Figure 4. CCDF of PAPR with different method to reduce the PAPR in IEEE 802.16e

In this part, the impact of the studied methods on CCDF will be discussed. The figure 4, shows the CCDF curves in function of $P A P R_{0}$ obtained by the different methods allowing the PAPR value reduction (TR, CL, TRCL) and the CCDF of a OFDM classic signal (i.e. without reduction technique of the PAPR "Original"). We can notice that the combinaison TRCL reduce the PAPR from $10.6 \mathrm{~dB}$ to $4.8 \mathrm{~dB}$ with a probability of approximately $10^{-2}$. It represents a reduction factor of about $5.8 \mathrm{~dB}$, while CL offers a reduction factor of approximately $5.5 \mathrm{~dB}$ and TR offers approximately $3 \mathrm{~dB}$. From that, we can conclude that the CCDF of the TRCL and CL methods are comparable and decrease rapidly compared to the others methods (TR and Original).

The second results shown in figure 5, represents the temporal evolution of the OFDM signal's envelope for the different PAPR reduction methods. These results are obtained from a unit mean power signal with a clipping of normalized amplitude $A=1.65$ to have a value of $P A P R_{- \text {target }}$ equal to2.17 (see equation 11).

$$
A=10^{\frac{P A P R_{- \text {target }}}{10}} \cdot E\left(|x(t)|^{2}\right)
$$

It can be noted that the TR (figure 5.a) method leaves a few peaks that exceed the $A$, the CL method 


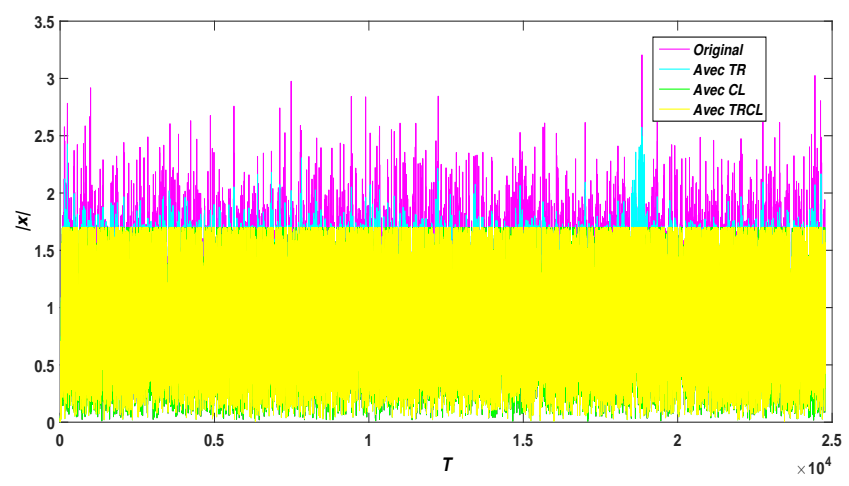

(a). With the different methods of the PAPR reduction.

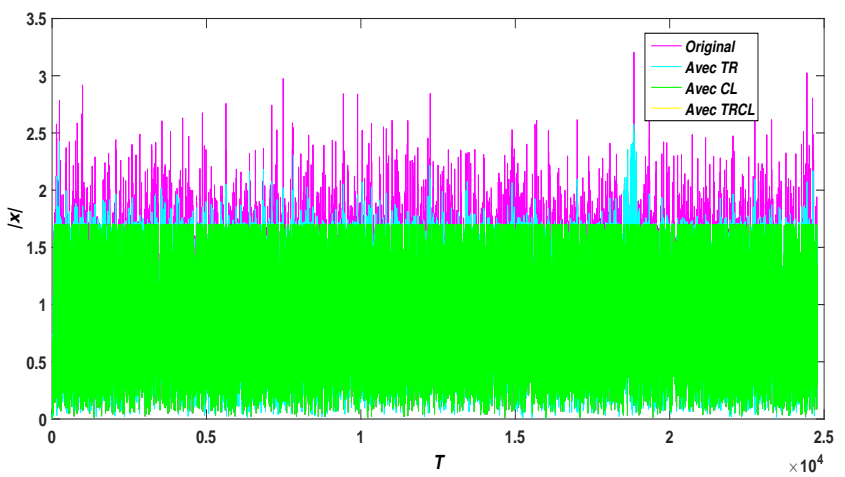

(b). Without TRCL.

Figure 5. "The temporal evolution of the envelope for OFDM signal."

(figure 5.b) clipped all the peaks that are higher than $A$, and for TRCL (figure 5.a) we can show that the results obtained are identical to those of CL, but with an improvement in BER and EVM transmission errors (as we will see later on).

In the next section, a nonlinear amplifier and a channel model will be introduced to observe the degradation of the CL method and the performance of our TRCL method in terms of BER and EVM.

\subsection{Performances in presence of nonlinear PA model}

The emission information in wireless networks over a great distance such as WIMAX requires the use of power amplifiers PA [3], to ensure a complete transmission. The role of PA is to increase the power of the RF signal without distorting the signal at its input and without energy dissipation when issued to its output. The following equation 12 represents the relationship non-linear between the input signal to a PA and its output:

$$
P_{\text {out }}=G\left(P_{\text {in }}\right) \cdot P_{\text {in }}
$$

where $P_{i n}$ and $P_{\text {out }}$ are the PA power of the input and the output signal, respectively, and $\mathrm{G}$ is the gain of the PA depending on the amplifier's input power. The gain value remains constant for the weak powers in the linear zone, it will be called also linear gain, then it decreases when the power increased in the other areas.

PA is characterized by two inputs-outputs relationships also called transfer characteristics of conversions AM/AM (Amplitude Modulation) and AM/PM (Phase Modulation). The AM/AM represents the variation of the output signal power depending on the input signal power (as show in figure 6), while the AM/PM represents the phase difference between the PA output and input signals depending on the input signal power. 


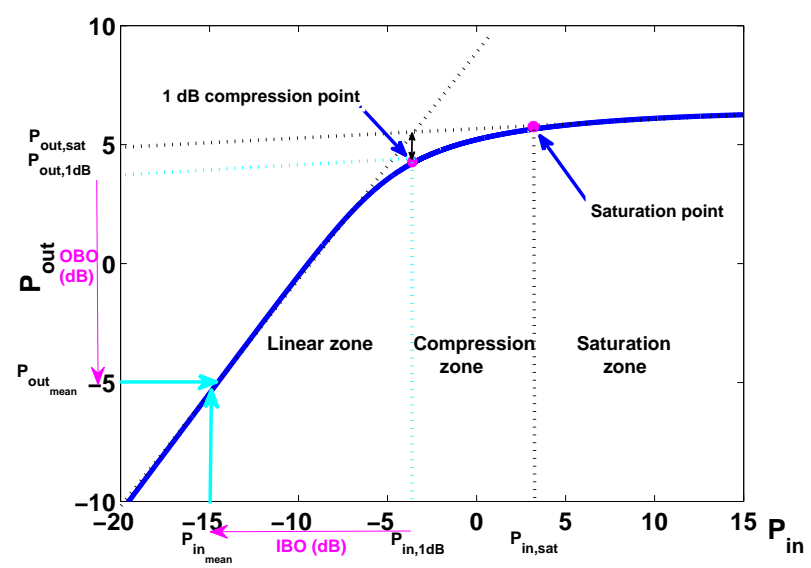

Figure 6. AM/AM characteristics

The mathematical model of the amplifier PA is based on the most widespread models, namely, the Rapp model described by the expression of conversion AM/AM (equation 13) and the Saleh model which is described by AM/PM (as show in equation 14) [23]:

$$
F_{\text {AMAM }}(|x(t)|)=\frac{|x|}{\left(1+\left(\frac{|x|}{A_{\text {sat }}}\right)^{2 p}\right)^{\frac{1}{2 p}}}
$$

where $|x(t)|$ is the instantaneous modulated envelope of the input signal. With $A_{\text {sat }}=2.1$ and $p=3$

$$
F_{A M P M}(|x(t)|)=\frac{\alpha_{p} \cdot|x|^{2}}{1+\beta_{p} \cdot|x|^{2}}
$$
amplifier.

with $\alpha_{p}=p i / 3$ and $\beta_{p}=1$. The parameters $\alpha_{p}$ and $\beta_{p}$ are used to describe the behavior of the

The AM / AM curve of the PA is divided into three functioning zones (According to figure 6), the linear zone of the amplifier where the output power is proportional to the input power according to a gain of the amplifier which remains constant. After a certain power input, the gain starts to decrease slowly, this zone is called compression zone. And finally, the saturation zone where the amplifier reaches its maximum output power. The point to $1 \mathrm{~dB}$ compression of gain represents the power output where the difference between the gain calculated in the linear zone and the real gain of the PA is worth than $1 \mathrm{~dB}$. The saturation point is a point where the amplifier reaches the maximum value to issue an output signal and its power appointed power of saturation. Most used amplifiers are non-linear, since the linear amplifiers are very expensive. However, the non-linearities of a PA has to impact the existence of distortions temporal and frequency signals to transmit which are reflected by a distortion of the signal.

To avoid such distortions, we must take a step back in power, compared to the point of compression to $1 \mathrm{~dB}$ steps. This decline allows to work in the linear zone as well as the signal is closest to the non-linear zone of the PA. It is defined by the IBO (Input Back-off) and the OBO (Ouput Back-off) which represents the decline in average power input and output, respectively, compared to the point of compression to $1 \mathrm{~dB}$ (figure 6).

In our simulations, we have added noise to simulate a Additive White Gaussian Noise channel (AWGN). This is a fundamental characteristic of the transmission channel. This channel noise is modeled by a random signal $n(t)$, whose probability distribution follows the Gaussian law. The received signal:

$$
r(t)=s(t)+n(t)
$$



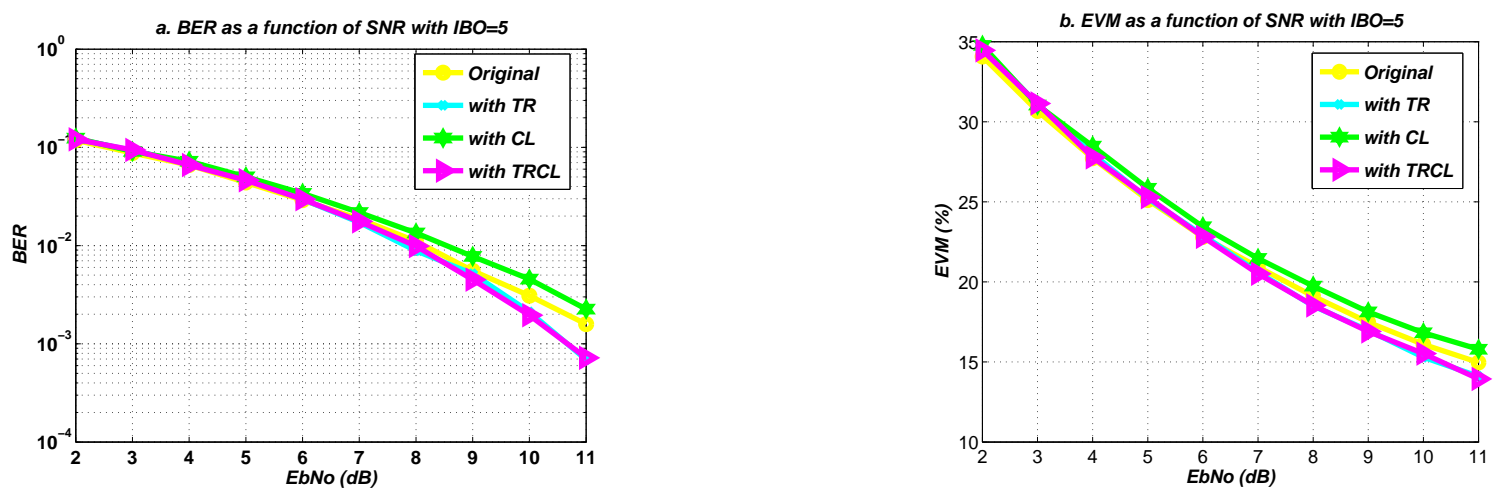

Figure 7. EVM and BER as a function of $\mathrm{SNR}$ with $\mathrm{IBO}=5$
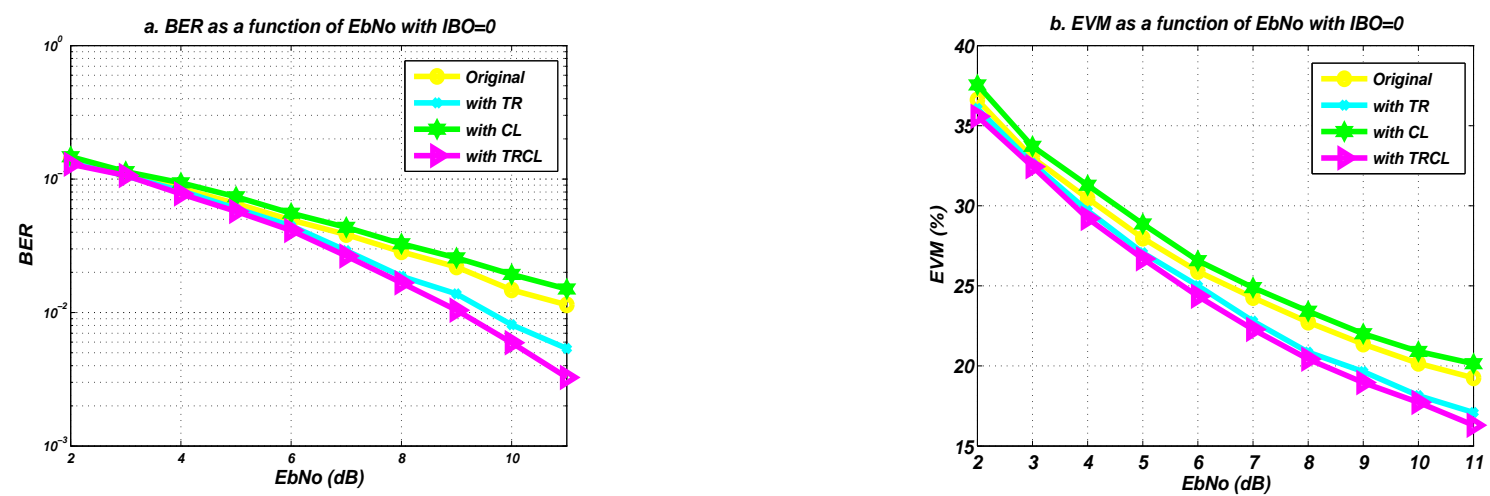

Figure 8. EVM and BER as a function of SNR with $\mathrm{IBO}=0$

where $\mathrm{s}(\mathrm{t})$ represents the transmitted signal.

After the addition of noise, we first calculated the percentage of Error Vector Magnitude (EVM) which corresponds to the error vector's amplitude between the origin constellation and the received constellation. Second, we calculated the Bit Error Rate (BER) which is the ratio between the number of errors bits at reception $(\mathrm{Ne})$ and the total number of transmitted bits $(\mathrm{N})$.

In the first figures (7.a and 7.b), we can show the evolution curves of the EVM and the BER according to the SNR $(\mathrm{Eb} / \mathrm{No})$ that varies between $2 \mathrm{~dB}$ and $11 \mathrm{~dB}$ for a backward movement of $I B O=5 \mathrm{~dB}$, to make a comparison between the various methods for PAPR reduction.

For the low SNR values $(S N R<7) \mathrm{dB}$, we can see that all BER and EVM curves have almost the same behavior. For strong SNR $(S N R>7) \mathrm{dB}$, a first remark concerns the Clipping method which degrades all communication criteria. We also see that of TR which coincides with TRCL with a non-negligible improvement of BER and with a slight improvement of EVM compared to the other methods.

After, the amplifier operates in the non-linear zone at the point at $1 \mathrm{~dB}$ of compression corresponding to a decrease in power of zero input $(I B O=0 \mathrm{~dB})$. The curves in Figure 8 show the comparison between the BER and the EVM according to SNR (Eb/No) varying between $2 \mathrm{~dB}$ and $11 \mathrm{~dB}$ to make a comparison between various methods which allows to reduce the value of PAPR. From the results of these figures, We can also see that the CL method degrades all the criteria and the TRCL method maintains a good improvement when the noise level exceeds $E b / N o=8 \mathrm{~dB}$. We can notice that our TRCL method always retains the best level of performance on both criterias.

In the following figure 9, we evaluated the transmission quality in terms of BER and EVM for each method by varying the value of the amplifier's input down IBO (IBO varies between $-3 \mathrm{~dB}$ and $2 \mathrm{~dB}$ in a step $1 \mathrm{~dB}$ ) with fixed SNR value $=12 \mathrm{~dB}$. We noted the improvements made by applying a clipping after the TR method. 

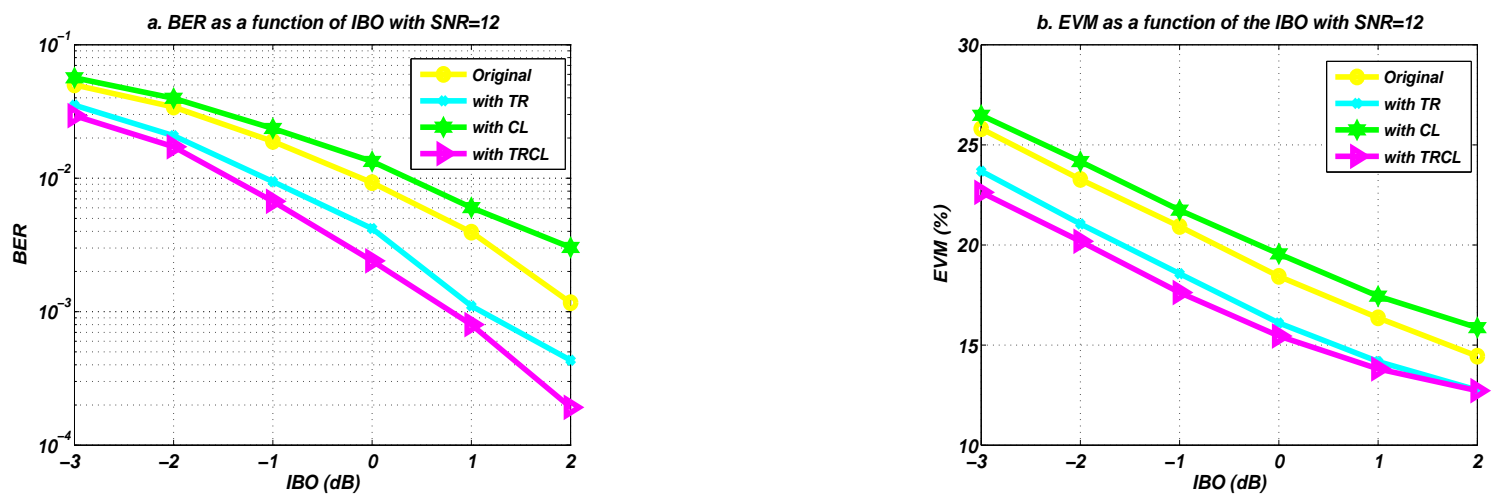

Figure 9. EVM and BER as a function of the IBO

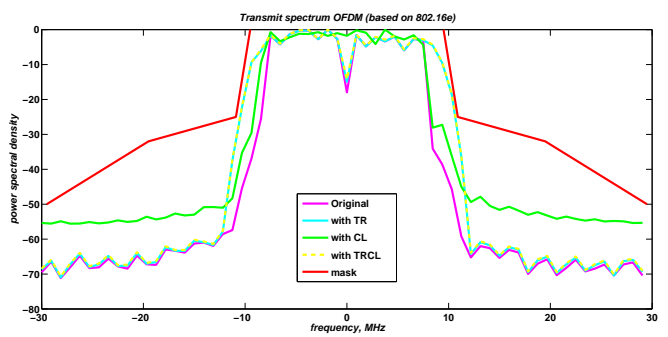

Figure 10. Transmit spectrum OFDM based on 802.16e without amplifier

Simulation results in figure 9.a show that when the IBO increases, the BER tends toward zero with the TRCL method. It is the proof that there is less disruption. In effect, an IBO low engenders significant distortions in the signal.

We can also observe in figure 9.b that TRCL improves EVM by about $3 \%$ when the IBO increases, that is to say that, when the amplification is done more and more in the linear area. However, low values of the IBO mean that the power amplifier operates on the limit of its saturation zone and will cause distortions in the amplified signal.

Figure 10 and 11 show the signal spectrum before and after the use of methods which reduce the PAPR, for a bandwidth of $30 \mathrm{MHz}$. We can see that the application of TR and TRCL method lead to a spectrum broadening of the signal, this is due to the use of the 64 free sub-carriers of the standard. It is verified on the spectra input and output of the amplifier.

In addition, at the output of the amplifier, we can see the spectral ascents in the bands due to nonlinear effects. However, these spectral lifts stay below the IEEE 802.16 standard mask.

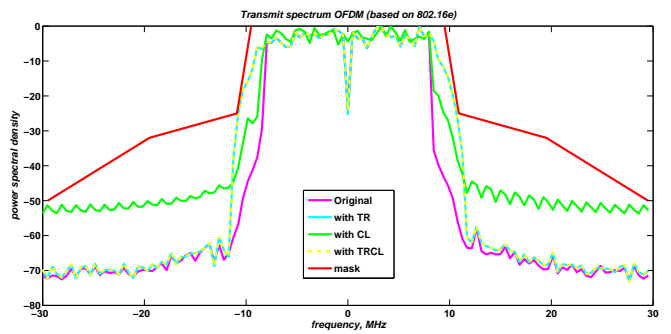

Figure 11. Transmit spectrum OFDM based on 802.16e with amplifier 


\section{Conclusion}

OFDM is one of the most popular physical layer technologies for high data rate wireless communications due to its robustness, high spectral efficiency and low computational complexity. The transmit signals in OFDM system can have high peak values in the time domain. Our observations about the TR method show that it is difficult due to the specificities of current standards to eliminate all high power peaks. The study presented in this article was used to evaluate the possibilities to associate a classic clipping, resulting in a good compromise between the backward compatibility of the TR method and best performances in PAPR reducing of the CL. The best performance of our TRCL technique in terms of PAPR reduction, the communication criteria EVM and BER on the WiMax standard allows to envisage an improvement in energy balance sheet of the amplification stage while maintaining a good transmission quality.

In addition, we measured the spectral of the signals at the input and at the output of the amplifier, of different methods which reduce the PAPR. Analysis of the results shows the improved performance of "TRCL" while respecting the specifications of the standard used. Indeed, we also found that the spectrum of the signals at the output of the amplifier meets to the mask imposed by the standard IEEE 802.16.

\section{REFERENCES}

[1] V. N. Richard and R. Prasad, "OFDM for Wireless Multimedia Communications," 1st ed. Artech House Publishers, Norwood, MA, USA, 2000.

[2] M. Deumal, A. Behravan, T. Eriksson, and J. L. Pijoan, "Evaluation of performance improvement capabilities of paprreducing methods," Wirel. Pers. Commun., vol. 47, no. 1, pp. 137-147, Oct. 2008.

[3] S. Bachir and C. Duvanaud, "New Identification Procedure for Continuous-Time Radio Frequency Power Amplifier Model," Circuits, Systems, and Computers, vol. 19, no. 06, pp. 1259-1274, 2010.

[4] N. Calinoiu, S. Bachir, and C. Duvanaud, "Mixed time and frequency-domain identification of radio frequency power amplifiers," Microwave Conference (EuMC), 2010 European, pp. 1018-1021, sept. 2010.

[5] A. Gatherer and M. Polley, "Controlling clipping probability in dmt transmission," Signals, Systems amp; Computers, 1997. Conference Record of the Thirty-First Asilomar Conference on, vol. 1, pp. 578-584, nov. 1997.

[6] T. Deepa and R. Kumar, "Papr reduction for ofdm systems using clipping and square rooting techniques," Proceedings of the International Conference on Advances in Computing, Communications and Informatics, ser. ICACCI '12, ACM publisher, New York, NY, USA, pp. 740-744, 2012.

[7] X. Li and J. Cimini, L.J., "Effects of clipping and filtering on the performance of OFDM," Vehicular Technology Conference, 1997, IEEE 47th, vol. 3, pp. $1634-1638$, may 1997.

[8] Y. Wang and Z. Luo, "Optimized iterative clipping and filtering for PAPR reduction of OFDM signals," Communications, IEEE Transactions on, vol. 59, no. 1, pp. 33 -37, january 2011.

[9] S. P. Yadav and S. Chandra Bera, "Papr reduction for improved efficiency of ofdm modulation for next generation communication systems," International Journal of Electrical and Computer Engineering (IJECE), vol. 6, pp. 2310-2321, oct 2016.

[10] Iram Maisarah Mokhtar, Norulhusna Ahmad, Hazilah Mad Kaidi, Mohd Azri Mohd Izhar and Norliza Mohamed, "PAPR Reduction Techniques in Generalized Inverse Discrete Fourier Transform Non-orthogonal Frequency Division Multiplexing System Iram," Indonesian Journal of Electrical Engineering and Computer Science, vol. 10, no. 3, pp. 1045-1052, June 2018.

[11] C.-L. Wang, S.-J. Ku, and C.-J. Yang, "A low-complexity PAPR estimation scheme for OFDM signals and its application to SLM-based PAPR reduction," Selected Topics in Signal Processing, IEEE Journal of, vol. 4, no. 3, pp. 637 -645 , june 2010.

[12] C.-E. Weng, C.-W. Chang, C.-H. Chen, and H.-L. Hung, "Novel low-complexity partial transmit sequences scheme for papr reduction in ofdm systems using adaptive differential evolution algorithm," Wirel. Pers. Commun., vol. 71, no. 1, pp. 679-694, Jul. 2013.

[13] A. Jones, T. Wilkinson, and S. Barton, "Block coding scheme for reduction of peak to mean envelope power ratio of multicarrier transmission schemes," Electronics Letters, vol. 30, no. 25, pp. 2098-2099, dec 1994.

[14] P. N. Kota , Ashwini Argade, Komal Kand and Shrutika Pote, "Tone Injection Method for Reduction of Peak-ToAverage-Power (PAPR) in OFDM System," International Journal of Electrical, Electronics and Computer Systems (IJEECS), vol. 5, pp. 2347-2820, 2017.

[15] I. Mahafeno, Y. Louet, and J.-F. Helard, "Peak-to-average power ratio reduction using second order cone programming based tone reservation for terrestrial digital video broadcasting systems," Communications, IET, vol. 3, no. 7, pp. 1250 -1261 , july 2009.

[16] P. Kangwoo and P. . In cheol, "Low-complexity tone reservation for papr reduction in OFDM communication sys- 
tems," Very Large Scale ., IEEE International Transactions, October 2012.

[17] B. Koussa, S. Bachir, C. Perrine, C. Duvanaud, and R. Vauzelle, "A comparison of several gradient based optimization algorithms for PAPR reduction in OFDM systems," CCCA ., The 2nd IEEE International conference, 2012.

[18] E. Polak and G. Ribire, "Note sur la convergence de méthodes de directions conjuguées," Rev. Fran. Informat. Rech. Opr, vol. 3, no. 1, pp. 35-43, 1969.

[19] B. Koussa, S. Bachir, C Perrine, C. Duvanaud, and R. Vauzelle, "Evaluation expérimentale d'une optimisation de la méthode TR pour la réduction du PAPR," 18èmes Journées Nationales Microondes, Paris, France, pp. 4, May 2013.

[20] C. So-In, R. Jain, and A.-K. Tamimi, "Capacity evaluation for ieee 802.16e mobile wimax," J. Comp. Sys., Netw., and Comm., vol. 2010, pp. 1:1-1:12, Jan. 2010.

[21] R. Jonathan Shewchuk, "An introduction to the conjugate gradient method without the agonizing pain," Technical report, Pittsburgh, PA, USA, 1994.

[22] Elijah, Polak, "Optimization: Algorithms and Consistent Approximations," Springer-Verlag New York, Inc., New York, NY, USA, 1997.

[23] H. Ku and J. Kenney, "Behavioral modeling of nonlinear rf power amplifiers considering memory effects," Microwave Theory and Techniques, IEEE Transactions on, vol. 51, no. 12, pp. 2495-2504, Dec 2003. 